\title{
Detectability of Earth mass planets with RV techniques around Sun-like stars. The example of the Sun
}

\author{
Anne-Marie Lagrange ${ }^{1}$, Nadege Meunier $^{1}$ and Morgan Desort ${ }^{1}$ \\ LAOG, Université J Fourier, F-38041 Grenoble Cedex, France \\ email: lagrange@obs.ujf-grenoble.fr, meunier@obs.ujf-grenoble.fr, \\ desort@obs.ujf-grenoble.fr
}

\begin{abstract}
We present the results of detailed simulations of the RV and astrometric signals expected from the Sun, when taking into account its activity (spots, plages, convection). To do so, we considered all structures $(2,000,000)$ identified on the Sun surface over a full cycle. We show that the Sun activity would prevent the detection of the Earth in the Habitable Zone with RV technics with today or future forthcoming instruments, mainly because of inhomogeneous convection. We also show that the activity-induced signal would be comparatively easier for the astrometric detection of the Earth of similar planets.
\end{abstract}

Keywords. planetary systems, Sun: activity, stars: activity, techniques: radial velocities, astrometry

\section{Introduction}

It has been known that stellar spots could impact on the detectability of extrasolar planets with radial velocities (RV) technics. The most detailed simulations have shown (Desort et al. 2007) that depending on their characteristics (size, location, temperature), and depending on the star properties (inclination), spots can produce RV variations quite similar to those induced by planets. Such spots represent a potential problem and are of growing importance as lower and lower mass planets are being (and will be) searched at both short and long periods with next generation RV instruments. In addition, other contrasted structures (plages, networks) or convection will impact the RV signal, but prior to the present study, no detailed estimation was available. To further investigate, in a realistic case, the impact of activity on the detection of Earth mass planets in the Habitable Zone (HZ), we have performed a complete simulation of the RV variations expected from the Sun, if observed like a star, during one entire, $11 \mathrm{yr}$ solar cycle. The results are presented below. We finally also address the same planet detectability, under similar conditions, with astrometric technics.

\section{Impact of activity on planet detection with RV technics}

Spots only (Lagrange et al. 2010a We considered all (160000) spots with sizes $\geqslant 1$ ppm identified each day on the Sun surface during one cycle (Jan 93-Dec 03) from Debrecen Observatory. Using this map, and assuming that the spots are $550 \mathrm{~K}$ cooler than the Sun surface, we computed the emerging spectrum and photometric signal that one would measure on the spectrum of the Sun if seen like a star with Teff $=5800 \mathrm{~K}$ and vsini $=1.9$ $\mathrm{km} / \mathrm{s}$. We then analysed the spectra as done in RV searches to extract the resulting RVs, Bissector Velocity Span temporal variations. These variations are then those expected from a solar type star with the same level of activity, seen edge-on. We then added 
$1 \mathrm{ME}$ planet in the $\mathrm{HZ}$ as well as instrumental noise $(1-5-10 \mathrm{~cm} / \mathrm{s})$ corresponding to that expected from the forthcoming RV instruments (Espresso, Codex).

It appears that during the high-activity period, the planet would not or hardly be detected from the corresponding periodograms of the RV variations. During low activity periods, it could be detected with the next generations of RV instruments provided very intensive and long surveys (star observed twice a week over several years).

Impact of solar spots and plages on planet detection (Meunier et al. 2010) We considered then both (20800) spots groups larger than $10 \mathrm{ppm}$ (NOAA data) and bright structures (plages and magnetic network; 1.803.344 structures from SOHO MDI magnetograms) observed between May 96 to Oct 07 . The spots and bright structures temperatures were deduced from the comparison between our simulated photometric data and the observed TSI data. The conclusions are similar to the spots-only case.

Impact of solar spots, plages and convection on planet detection (Meunier et al. 2010) We finally considered in addition the impact of inhomogeneous convection. To do so, we assumed that the quiet Sun (ie without spots/bright structures) has a convective blueshift of $200 \mathrm{~m} / \mathrm{s}$, and in a first step, that this convective blueshift is canceled within the spots, plages and network. It appears that convection induced RV signal dominates that of the spots and plages over the whole cycle (Table 1). With the knowledge and tools available today, an Earth mass planet orbiting the Sun in the HZ would not be detected with the next generation instruments, even with a daily temporal sampling. A slightly different (e.g. 2/3) attenuation of the convective blueshift instead of a total one would not change the present results.

Table 1. Measured RMS for the shifts along and perpendicular to the equatorial plane (in $\mu$ as) due to the spots and bright structures. The entire cycle as well as low and high activity periods are considered. Associated rms of the RV (m/s), taking or not convection into account, as well as photometry (relative variations) are also given.

\begin{tabular}{llllll}
\hline & $\mathrm{rms}($ deltax $)$ & $\mathrm{rms}($ deltay $)$ & $\mathrm{rms}(\mathrm{RV}$ without conv. $)$ & $\mathrm{rms}(\mathrm{RV}$ with conv. $)$ & $\mathrm{rms}(\mathrm{TSI})$ \\
all & 0.07 & 0.05 & 0.33 & 2.4 & $3.6 \mathrm{e}-4$ \\
high & 0.09 & 0.06 & 0.42 & 1.42 & $4.5 \mathrm{e}-4$ \\
low & 0.02 & 0.01 & 0.08 & 0.44 & $1.2 \mathrm{e}-4$ \\
\hline
\end{tabular}

\section{Astrometric detection}

Observables which are not sensitive to convection (photometry, astrometry) represent an attractive solution to search for Earth mass planets in the HZ. Using the same spots+bright structures simulations as described above, we have simulated the resulting astrometric variations. It appears that the Earth astrometric signal (amplitude $0.3 \mu \mathrm{as}$ ) dominates the activity one ( $\mathrm{rms} \leqslant 0.1 \mu \mathrm{as}$; Table 1 ), and that a monthly monitoring of the astrometric position over about 4 years would allow the detection of an Earth in the $\mathrm{HZ}$, provided instrumental precisions of the order of 1 . $\mu$ as per measurement (Lagrange et al. 2010b).

\section{References}

Desort, M., Lagrange, A.-M., Galland, F, et al. 2007, A\&SA, 473, 893

Lagrange, A.-M., Desort, M., \& Meunier, N. 2010, A\& $A$, 512, A38

Lagrange, A.-M., Meunier, N., Desort, M., \& Malbet, F. 2011, A\&A, 528, L9

Meunier, N., Desort, M., \& Lagrange, A.-M. 2010, A\&GA, 512, A39 